\title{
Erratum to: Development of polymorphic microsatellite markers \\ for the microendemic pupfishes Cyprinodon julimes and $C$. pachycephalus
}

Evan W. Carson ${ }^{1} \cdot$ Rochelle R. Beasley $^{2} \cdot$ Kenneth L. Jones $^{3} \cdot$ Stacey L. Lance $^{2}$ • Ma de Lourdes Lozano-Vilano ${ }^{5}$ Lilia Vela-Valladares ${ }^{4}$ - Iris Banda-Villanueva ${ }^{4}$. Thomas F. Turner ${ }^{1} \cdot$ Mauricio De la Maza-Benignos ${ }^{4}$

Published online: 7 May 2015

(C) Springer Science+Business Media Dordrecht 2015

Erratum to: Conservation Genet Resour (2013) 5:853-856

DOI 10.1007/s12686-013-9925-5

Unfortunately, in the original publication, the DNA sequences of the forward primers (all loci) in Table 1 were published incorrectly. The revised Table 1 given below contains the corrected sequences for all primers.

The online version of the original article can be found under doi:10. 1007/s12686-013-9925-5.

\section{Evan W. Carson}

evan.carson@gmail.com

1 Department of Biology and Museum of Southwestern Biology, University of New Mexico, Albuquerque, NM 87131, USA

2 Savannah River Ecology Laboratory, University of Georgia, Aiken, SC 29802, USA

3 Department of Biochemistry and Molecular Genetics, University of Colorado School of Medicine, Aurora, CO 80045, USA

4 Pronatura Noreste, A.C, Loma Larga 235, Col. Loma Larga, 64710 Monterrey, NL, Mexico

5 Laboratorio de Ictiología, Facultad de Ciencias Biológicas, Universidad Autónoma de Nuevo León, 66450 San Nicolás de los Garza, NL, Mexico 
Table 1 Details for 28 polymorphic microsatellite loci developed for Cyprinodon julimes

\begin{tabular}{|c|c|c|c|c|c|c|c|c|c|}
\hline Locus & Primer sequence $5^{\prime} \rightarrow 3^{\prime}$ & Repeat motif & Size (bp) & $\mathrm{N}$ & $K$ & $H_{O}$ & $H_{E}$ & PI & $\mathrm{TD}$ \\
\hline \multirow[t]{2}{*}{ Cyju1 } & F: *TTTCAGCGGCCCTTATGC & ATCT & $318-362$ & 17 & 6 & $0.176^{\dagger}$ & 0.777 & 0.085 & 65 \\
\hline & R: AAAGATACGTCAGCACCGCC & & 319-395 & 20 & 10 & $0.300^{\dagger}$ & 0.670 & 0.126 & 65 \\
\hline \multirow[t]{2}{*}{ Cyju 2} & F: *CCCAATCACCCATAGACTGG & ATGG & $235-279$ & 17 & 4 & $0.118^{\dagger}$ & 0.621 & 0.203 & 65 \\
\hline & R: AAGAAATGTGGACACAGCATCC & & NA & & & & & & \\
\hline \multirow[t]{2}{*}{ Сујиз } & F: *GAAAGGTACTAGTCAGTTGTGTTACTGG & ATGG & $369-389$ & 19 & 5 & 0.789 & 0.720 & 0.126 & 65 \\
\hline & R: AATCTCATATCGGCTCACGC & & $330-358$ & 23 & 6 & 0.783 & 0.766 & 0.087 & 65 \\
\hline \multirow[t]{2}{*}{ Суju4 } & F: *CGGGAATGCGTCTCTTGG & ATGG & $213-249$ & 19 & 4 & 0.105 & 0.411 & 0.396 & 65 \\
\hline & R: ACCGTGACATGGACTAAGCG & & $246-262$ & 23 & 5 & 0.609 & 0.632 & 0.205 & 65 \\
\hline \multirow[t]{2}{*}{ Cyju5 } & F: *CCAATCATGTGCTTGCATCC & ATGG & $285-333$ & 19 & 8 & 0.789 & 0.760 & 0.091 & 65 \\
\hline & R: AGCATTTAAACTTCAAGCTGACGC & & $280-304$ & 23 & 7 & 0.826 & 0.767 & 0.090 & 65 \\
\hline \multirow[t]{2}{*}{ Суju6 } & F: *GTTTACATGAAATAAGGAGGTGTAAGC & ATCT & $248-320$ & 19 & 14 & 0.947 & 0.884 & 0.024 & 65 \\
\hline & R: AGCTGCTAGCTGGGTGCC & & NA & & & & & & \\
\hline \multirow[t]{2}{*}{ Суји10 } & F: *TTTGTATCTTGTCTTATCCCTTGC & ATGG & $398-414$ & 16 & 5 & 0.813 & 0.658 & 0.152 & 65 \\
\hline & R: CACCTAAATAACACAACTAATGCCC & & $193-213$ & 23 & 5 & 0.565 & 0.680 & 0.140 & 65 \\
\hline \multirow[t]{2}{*}{ Суju11 } & F: *TATCGCCCTAATATCCGTCC & ATGG & $375-399$ & 19 & 5 & 0.684 & 0.760 & 0.096 & 65 \\
\hline & R: CACTCTGAGCCAATCCAACC & & NA & & & & & & \\
\hline \multirow[t]{2}{*}{ Суји12 } & F: *GGGCTTAGGTGACTTGGTGC & AAAT & $210-258$ & 19 & 7 & 0.579 & 0.795 & 0.068 & 65 \\
\hline & R: CAGACTTTGACTAGGCCATTTCC & & $215-227$ & 23 & 3 & 0.522 & 0.581 & 0.251 & 65 \\
\hline \multirow[t]{2}{*}{ Суји13 } & F: *TTAGGGTTAGCTAATTCAACCTGC & AAAG & NA & & & & & & \\
\hline & R: CATAGGTGCTGTTTACCGCC & & $245-301$ & 22 & 8 & $0.136^{\dagger}$ & 0.517 & 0.251 & 65 \\
\hline \multirow[t]{2}{*}{ Суји15 } & F: *CATAGAAGTTTGGGTTGGAAGG & ATGG & $367-387$ & 19 & 4 & 0.737 & 0.675 & 0.172 & 65 \\
\hline & R: CCACATTCGCTAAGGTCTGG & & NA & & & & & & \\
\hline \multirow[t]{2}{*}{ Суји16 } & F: *CATGCTATCCAACACAAACATCC & ATGG & $193-221$ & 19 & 5 & 0.737 & 0.705 & 0.138 & 65 \\
\hline & R: CCCTGAGCCCACTGATAACC & & NA & & & & & & \\
\hline \multirow[t]{2}{*}{ Суји17 } & F: *GATATAATTATTTATTCACCAGGTGCG & AATAG & $328-343$ & 19 & 4 & 0.579 & 0.591 & 0.252 & 65 \\
\hline & R: CCTGTCAGGGTCTGAGAAAGC & & $324-344$ & 23 & 5 & $0.261^{\dagger}$ & 0.670 & 0.167 & 65 \\
\hline \multirow[t]{2}{*}{ Cуju18 } & F: *GCCCTGTATATGCTGAATTTACCC & AATC & $222-242$ & 19 & 5 & $0.474^{\dagger}$ & 0.694 & 0.134 & 65 \\
\hline & R: CCTGTCTTTAATAAGTTCTCTGTGGC & & $215-243$ & 23 & 7 & $0.261^{\dagger}$ & 0.817 & 0.058 & 65 \\
\hline \multirow[t]{2}{*}{ Суји 20} & F: *GAGACAGGAAACAGCACCTGG & ATGG & NA & & & & & & \\
\hline & R: CGTCACAAGGATACAATATGATGC & & $238-254$ & 23 & 5 & 0.870 & 0.750 & 0.106 & 65 \\
\hline \multirow[t]{2}{*}{ Суји 21} & F: *GACAGGTTTATTTGGTGGATGC & AATG & $304-336$ & 19 & 6 & 0.737 & 0.687 & 0.141 & 65 \\
\hline & R: CTCTGCCCTAACCACTCCC & & NA & & & & & & \\
\hline \multirow[t]{2}{*}{ Суju22 } & F: *TCAGGCAGCAACAAAGTTGG & ATGG & $301-369$ & 18 & 12 & 0.778 & 0.890 & 0.022 & 65 \\
\hline & R: CTTGGGCAGTTCTTCAGTGC & & NA & & & & & & \\
\hline \multirow[t]{2}{*}{ Суju 28} & F: *GGGTGCCAATCATCCATCC & ATGG & NA & & & & & & \\
\hline & R: GGAAACGTTGGGAAACTGGG & & $285-345$ & 22 & 5 & $0.273^{\dagger}$ & 0.593 & 0.221 & 65 \\
\hline \multirow[t]{2}{*}{ Суju29 } & F: *AAGAAAGAGATGCTGTGACTGTGG & ATGG & $328-368$ & 18 & 7 & 0.722 & 0.735 & 0.100 & 65 \\
\hline & R: GGATCTAAGCAATCTGCATATACAAGG & & $305-349$ & 23 & 11 & $0.261^{\dagger}$ & 0.888 & 0.022 & 65 \\
\hline \multirow[t]{2}{*}{ Сујиз1 } & F: *CCAAGGAGACCCAGAGGG & ATGG & $208-228$ & 19 & 2 & 0.421 & 0.499 & 0.375 & 65 \\
\hline & R: GTGGTACCAATCAAAGATTCAAAGG & & $172-192$ & 23 & 5 & $0.391^{\dagger}$ & 0.716 & 0.130 & 65 \\
\hline Сујиз2 & F: *GACGTACCCTGCCTCTCACC & ATGG & $212-248$ & 19 & 7 & 0.737 & 0.722 & 0.112 & 65 \\
\hline & R: TCAAGGCATTAAAGTATAGCTTGGC & & $209-249$ & 22 & 5 & 0.727 & 0.718 & 0.125 & 65 \\
\hline Сујиз3 & F: *GAACTTTGGTTTAACAAGCTGTCC & ATGG & $138-158$ & 19 & 4 & $0.158^{\dagger}$ & 0.240 & 0.587 & 65 \\
\hline & R: TCAATCCAGAAGTTAGGATAAATACG & & $119-139$ & 20 & 3 & 0.150 & 0.141 & 0.742 & 65 \\
\hline Сујиз4 & F: *ACGATCTCAGATTTCGCTGC & ATGG & $346-394$ & 19 & 7 & 0.842 & 0.783 & 0.080 & 65 \\
\hline & R: TCAGAGAATTGCTAAATCAAATGC & & $286-362$ & 23 & 6 & 0.609 & 0.674 & 0.137 & 65 \\
\hline Суju43 & F: *TTTGTTTACGCCCACTCACC & ATAGT & $298-318$ & 19 & 5 & 0.579 & 0.683 & 0.154 & 65 \\
\hline & R: TGGTCAGTTGTATGTTGAATGTTTACC & & $300-340$ & 23 & 7 & 0.739 & 0.811 & 0.063 & 65 \\
\hline
\end{tabular}


Table 1 continued

\begin{tabular}{|c|c|c|c|c|c|c|c|c|c|}
\hline Locus & Primer sequence $5^{\prime} \rightarrow 3^{\prime}$ & Repeat motif & Size (bp) & $\mathrm{N}$ & $K$ & $H_{O}$ & $H_{E}$ & PI & TD \\
\hline \multirow[t]{2}{*}{ Суји 45} & F: *CCAGTCATATGAATCGAAATTGACC & ATCT & $255-323$ & 19 & 12 & 0.737 & 0.799 & 0.055 & 65 \\
\hline & R: TGTCATCAGGTGCAGAAGTCC & & $231-283$ & 22 & 11 & 0.818 & 0.857 & 0.035 & 65 \\
\hline \multirow[t]{2}{*}{ Суju 46} & F: *GGCCTCAAAGTGGTCATTCC & ATCT & $276-328$ & 19 & 12 & 0.474 & 0.871 & 0.027 & 65 \\
\hline & R: TGTCATTTGCCACACCTTGC & & NA & & & & & & \\
\hline \multirow[t]{2}{*}{ Суju47 } & F: *TTGTCAACATCTCTTAATTTGTTTGC & ATGG & $257-265$ & 19 & 3 & 0.842 & 0.622 & 0.214 & 65 \\
\hline & R: TTTACACAAATACTGATCACTAGTCCC & & $227-267$ & 23 & 8 & 0.826 & 0.802 & 0.062 & 65 \\
\hline \multirow[t]{2}{*}{ Суju 48} & F: *TGAGACAGGCGACAATTCC & ATCT & $240-272$ & 18 & 8 & 0.556 & 0.744 & 0.103 & 65 \\
\hline & R: TTTCTGGTTTCTAGAGTTAATGCC & & $279-371$ & 23 & 14 & 0.826 & 0.854 & 0.034 & 65 \\
\hline
\end{tabular}

For each locus, the information in the top row refers to samples of $C$. julimes (Julimes pupfish), and the information in the second row refers to samples of $C$. pachycephalus (Bighead pupfish). Size is the range in length, in base pairs (bp), of observed alleles and includes the length of the CAG tag; $N$ is number of individuals genotyped; $k$ is number of alleles; $H_{O}$ is observed heterozygosity; $H_{E}$ is gene diversity; PI is probability of identity; TD refers to the touchdown protocol used for PCR (see text)

* CAG tag (5'-CAGTCGGGCGTCATCA-3')

$\dagger$ Significant deviation from Hardy-Weinberg proportions after Bonferroni corrections 